\title{
Nanocarriers for siRNA delivery to overcome cancer multidrug resistance
}

\author{
MENG QingShuo, YIN Qi \& LI YaPing* \\ Center of Pharmaceutics, Shanghai Institute of Materia Medica, Chinese Academy of Sciences, Shanghai 201203, China
}

Received March 29, 2013; accepted June 8, 2013

\begin{abstract}
Multidrug resistance (MDR) is an extremely complexed phenomenon in tumor chemotherapy and is one of the major obstacles for successful treatment. Discovery of RNA interference (RNAi) offers a new strategy with great potential to reverse MDR. Specific genes, which contribute to the emerging of MDR, can be silenced by RNAi. However, many natural barriers have to be overcome for efficient and safe delivery of siRNA. In recent years, the various kinds of nanocarriers, such as liposomes, cationic polymers, and inorganic materials, have been developed to deliver siRNA and show good results in reversing MDR. This review mainly summarizes the barriers in siRNA delivery and recent progress in designing nanotechnology-based siRNA delivery systems to overcome tumor MDR.
\end{abstract}

cationic polymers, inorganic carriers, liposomes, multidrug resistance, nanocarriers, siRNA delivery

Citation: $\quad$ Meng Q S, Yin Q, Li Y P. Nanocarriers for siRNA delivery to overcome cancer multidrug resistance. Chin Sci Bull, 2013, 58: 4021-4030, doi: 10.1007/ s11434-013-6030-9

Malignant tumor is one of the major diseases seriously affecting human health and threatening human life. World Health Organization (WHO) estimated that the number of deaths from cancer worldwide will exceed 13 million in 2030 (http://www.who.int/mediacentre/factsheets/fs297/en/ index.html). How to optimize the therapy for curing cancers has become an urgent problem to be solved. The chemotherapy is an important treatment option for patients with cancer. One of the major hurdles for successful chemotherapy is the eventual development of multidrug resistance (MDR). MDR, which can be intrinsic or acquired, is the phenomenon whereby exposure to one drug induces crossresistance to a variety of agents to which the cell has not been exposed [1]. The main mechanisms involved in tumor MDR contain increased drug efflux, decreased drug influx, activation of detoxifying systems (cytochrome P450), activation of DNA repair and blocked apoptosis (e.g. decreased ceramide levels) [2]. Overexpression of ATP-binding cassette (ABC) transporters, including MDR1 (ABCB1), MRP1

*Corresponding author (email: ypli@ simm.ac.cn)
(ABCC1) and ABCG2, was considered as the most common form. Detailed informantion about MDR has been expounded in some literature $[3,4]$.

A promising strategy to overcome MDR is to use RNA interference (RNAi) to selectively silence various oncogenes, turning off some related molecular pathways. RNAi is a sequence-specific and post-transcriptional gene silencing mechanism, in which small interfering RNA (siRNA) molecules (about 20-25 base pairs long) are involved [5]. Upon entry of double-stranded siRNA molecules into cells, the siRNA is activated through binding to an RNA-induced silencing complex (RISC), which unwinds the siRNA duplex and produces a single-stranded RNA targeted to complementary messenger RNA (mRNA) and finally the targeted mRNA is cleaved and destroyed [6,7]. Most of studies has shown that MDR phenotype is able to be reversed successfully with the help of RNAi. However, naked siRNA molecules usually have poor transfection efficiency, especially in vivo, due to its poor stability, low cellular uptake, and rapid clearance from the circulation [8]. Therefore, siRNA carriers, which can protect siRNA from degradation, 
deliver it to targeted tumor cells, increase its cellular uptake and finally silence the expression of targeted genes, are in urgent need.

Comparing with traditional viral carriers, non-viral ones are safer and have minimal immunogenicity. They are easy to be prepared on a large scale and at low cost $[9,10]$. Advances in nanotechnology open up unprecedented opportunities for obtaining excellent non-viral carriers. Nanocarriers such as liposomes, various kinds of cationic polymers, and inorganic materials have been developed to deliver siRNA and show good results in reversing MDR. This review mainly summarized recent progress in the designing of the siRNA delivery systems (SDS) and their applications to overcome tumor MDR.

\section{Barriers in siRNA delivery}

SDS is usually administrated intravenously or intraperitoneally in the treatment of multidrug resistant cancer [11]. For successful silence of targeted genes, there are mainly three barriers needing overcoming: stability in the blood circulation, uptake by targeted tumor cells and the arrival of siRNA in cytoplasm (Figure 1).

Firstly, nanocarriers should protect siRNA from degradation by nucleases and clearance by the reticuloendothelial system (RES) and avoid immune system recognition in the blood circulation [12]. The surface charge of the carrier is very important $[13,14]$. Cationic siRNA complexes could interact with negatively charged serum proteins such as serum albumin, lipoproteins or IgG proteins by non-specific electrostatic attraction, leading to increased size of the complexes and less siRNA accumulation in the targeted site.
Appropriate long circulation time, which can be realized by surface modifications of neutral hydrophilic polymers such as PEG, is desired in order to make full use of the enhanced permeability and retention effect (EPR). Secondly, after the SDS reached the targeted tissues, they are needed to be internalized by the targeted tumor cells. SDS can enter the cells by endocytosis pathway, and this process is influenced by the size and composition of the delivery system $[15,16]$. Modification by ligands, small molecule inhibitors, peptides and/or antibodies can be utilized to promote the entrance of SDS into tumor cells [17-20]. Finally, the complexes are usually transported to early endosomes with low $\mathrm{pH}$ environment after endocytosis. Then the early endosomes gradually evolved into a more acidic late endosomes and finally fused with lysosomes [21]. Complete siRNA with biological activity should be reserved in this process. The SDS or siRNA released from the vectors should have the ability to escape from endo/lysosomes and reach the intracellular targeted site, and siRNA must be freed into the cytoplasm timely. Only fully dissociated siRNA can form RISC and destroy the targeted mRNA. $\mathrm{pH}$ or reduction responsive materials can be utilized as siRNA carriers to improve this process. In a word, the ideal carriers should protect siRNA from various adverse factors that may damage the biological activities of siRNA in the previous procedures and release siRNA into cytoplasm in time.

\section{Nanocarriers for siRNA}

\subsection{Liposomes}

Among nonviral carriers, liposome is one of the most promising candidates for siRNA delivery [22]. Liposomes used

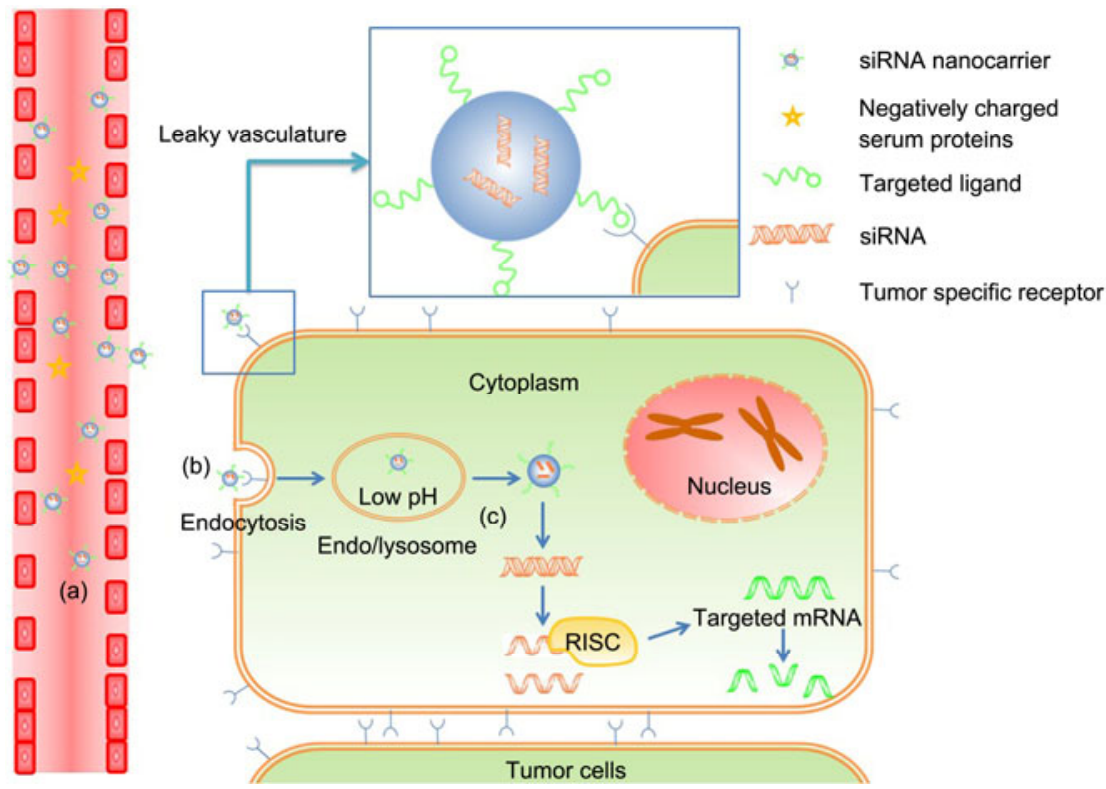

Figure 1 Three barriers in siRNA delivery. (a) Stability in the blood circulation; (b) uptake by targeted tumor cells via endocytosis pathway; (c) arrival of siRNA in cytoplasm. 
as siRNA vectors are different from the traditional ones with negative surface charge. Phospholipid that constitutes them usually contains different types of amine groups. In the formulations, these amino groups will carry positive charges after protonated. So they can form stable complexes with negatively charged siRNA through electrostatic interaction and deliver siRNA to targeted tissues or cells.

Cationic liposomes have been widely investigated. Several cationic lipids have been applied for siRNA interference, e.g. 1,2-dioleoyl-3-trimethylammonium-propane, $\mathrm{N}$-[1(2,3-dioleoyloxy)propyl]- $N, N, N$-trimethyl-ammonium methyl sulphate and $3 \beta-[N-(N, N$-dimethylaminoethane $)$-carbamoyl $]$ cholesterol. Saad et al. [23] developed the cationic liposome that can co-deliver doxorubicin (Dox), siRNA targeting MRPl mRNA as a suppressor of drug-efflux pumps and siRNA targeting Bcl2 mRNA as a suppressor of cellular anti-apoptotic defense (Figure 2). It increased the efficacy of Dox against multidrug resistant lung cancer cells by two different ways and achieved a synergy effect. In the study of Chen et al. [24], a multifunctional cationic liposome loaded with vascular endothelial growth factor (VEGF) siRNA and Dox was formed with a guanidinium-containing cationic lipid. With the help of guanidinium it can induce reactive oxygen species to inhibit the activity and expression of MDR transpoters. A synergistic effect was achieved with VEGF siRNA to overcome MDR. However, rapid clearance from the circulation and nonspecificity of cationic liposomes limited their in vivo applications. Some modified cationic liposomes were developed to address these problems. Polyethylene glycol (PEG)-coated Bcl2 siRNA-lipoplex was prepared by Nakamura et al. [25] to downregulate $\mathrm{Bcl} 2$ expression in the 5-Fluorouracil (5-FU) resistant DLD-1 cell line. Combined with treatment of $\mathrm{S}-1$, an oral formulation of Tegafur, a prodrug of 5-FU, it showed superior tumor growth suppression effect in the DLD-1 xenograft model than any single treatment. Modification with PEG prolonged circulation time of the lipoplex, decreased its immune stimulation and enhanced cellular uptake of the siRNA. Jiang et al. [26] developed a RGD peptide (arginine-glycine-aspartic acid)-modified cationic liposomes to specially deliver MDR1 siRNA to tumor cells. The RGD peptide could bind with integrin receptors, which were over-

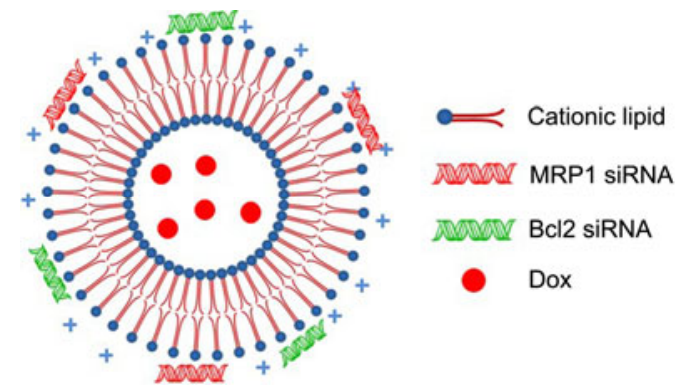

Figure 2 Cationic liposome co-delivering Dox, MRP1siRNA and Bcl2SiRNA. expressed on tumor cells, therefore increasing the accumulation of liposomes in tumors. The MDR1 siRNA complexes reversed MDR and significantly enhanced the effect of Dox in a mouse model of drug-resistant MCF7/ADR tumor by downregulating the expression of P-glycoprotein (P-gp) in tumor cells.

Compared with cationic liposomes, neutral liposomes, which are usually formed by 1,2-dioleoyl-sn-glycero-3-phosphatidylcholine (DOPC), usually present much lower cytotoxicity. Halder et al. [27] developed a neutral liposomal system to deliver Focal adhesion kinase (FAK) siRNA. FAK plays an important role in tumor growth and is predictive of poor clinical outcome in ovarian cancer. The complex is highly effective for down-regulating FAK expression in chemotherapy-resistant HeyA8MDR and A2780CP20 nude mice models. Combined with its treatment, the effect of docetaxel or cisplatin on chemotherapy-resistant tumors was enhanced by both direct and indirect antiangiogenic effects.

\subsection{Cationic polymers}

Cationic polymers receive much attention due to their good stability, simple preparation and easily modified chemical structures [28]. There have been several kinds of cationic polymers reported to deliver siRNA, such as poly (ethylene imine) (PEI), poly (amido amine) (PAMAM), polyamino acid (PAA), poly ( $\beta$-amino esters) (PAE), chitosan and so on.

(1) PEI. PEI is one of the most extensively investigated non-viral vectors [29]. The high content of amino groups in PEI enables itself to condense negatively charged siRNA firmly. PEI can exert proton sponge effect in the endosomes and release the intact siRNA into cytosol where it can initiate the RNA interference pathway [30]. Branched PEI 25 ( $\mathrm{kDa})$ has been considered the "golden" standard of nonviral gene delivery system because of its high transfection efficacy [31]. A common problem with PEI is its severe cytotoxicity and poor compatibility [32-34]. The strong positive surface charge of PEI is considered to be the main factor associated toxicity. It has been shown that the toxicity of PEI is strongly correlated with its molecular weight (MW) as well as its structure (branched or linear) [35]. Lowmolecular-weight PEI $(<5 \mathrm{kDa})$ usually show acceptable toxicity. But their efficiency to deliver siRNA is poor. As a result, synthesis of a modified PEI with low cytotoxicity and enhanced RNAi efficiency has become the recent research focus.

A lipid-substituted low molecular weight $(2 \mathrm{kDa})$ PEI was reported by Aliabadi et al. [36] to deliver breast cancer resistance protein (BCRP) siRNA. The complexes constituted by BCRP siRNA and lipid-substituted PEI downregulate BCRP expression and sensitize the drug-resistant cells to chemotherapy drug mitoxantrone, a substrate of $\mathrm{BCRP}$, by a 14-fold decrease in the $\mathrm{IC}_{50}$ value. Xue and 
Wong [37] developed a solid lipid PEI hybrid nanocarrier to deliver survivin-targeting siRNA (Figure 3(a)). Knockdown of survivin contributed to sensitize drug-resistant cancers to chemotherapy compound. This hybrid nanocarrier was cancer-specific by coating with folate ligand and realized sustained intracellular siRNA release. An extended RNAi effect was observed in in vivo experiment. In Xue and Wong's
[38] work, PEI hybrid nanocarriers decorated with apolipoprotein $\mathrm{E}$, a ligand targeting the surface endocytotic receptor megalin, were developed for specific delivering clusterin siRNA, which enhanced the transfection specificity of PEI. Knockdown of clusterin improved chemosensitization to subsequent paclitaxel treatment. Besides lipid, phospholipid was also used to improve the RNAi efficacy of PEI. Navarro

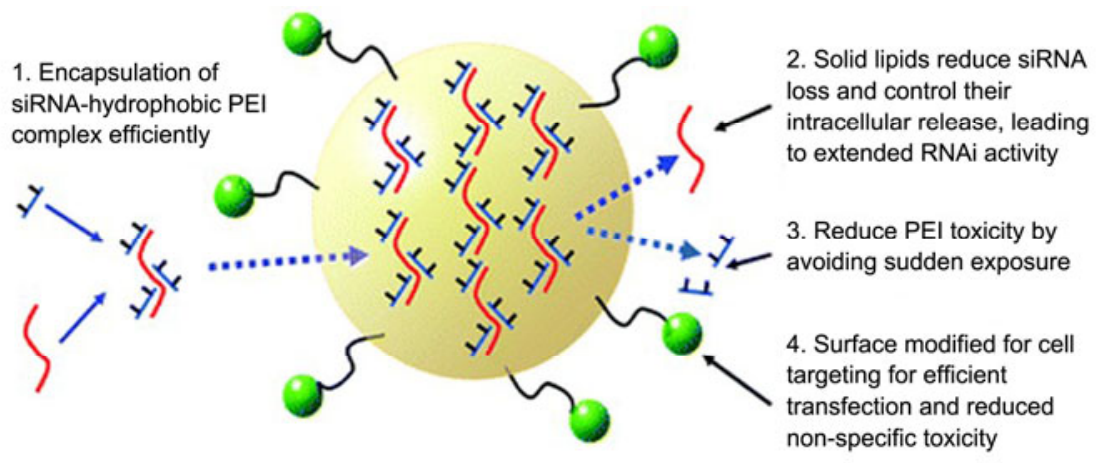

(a)
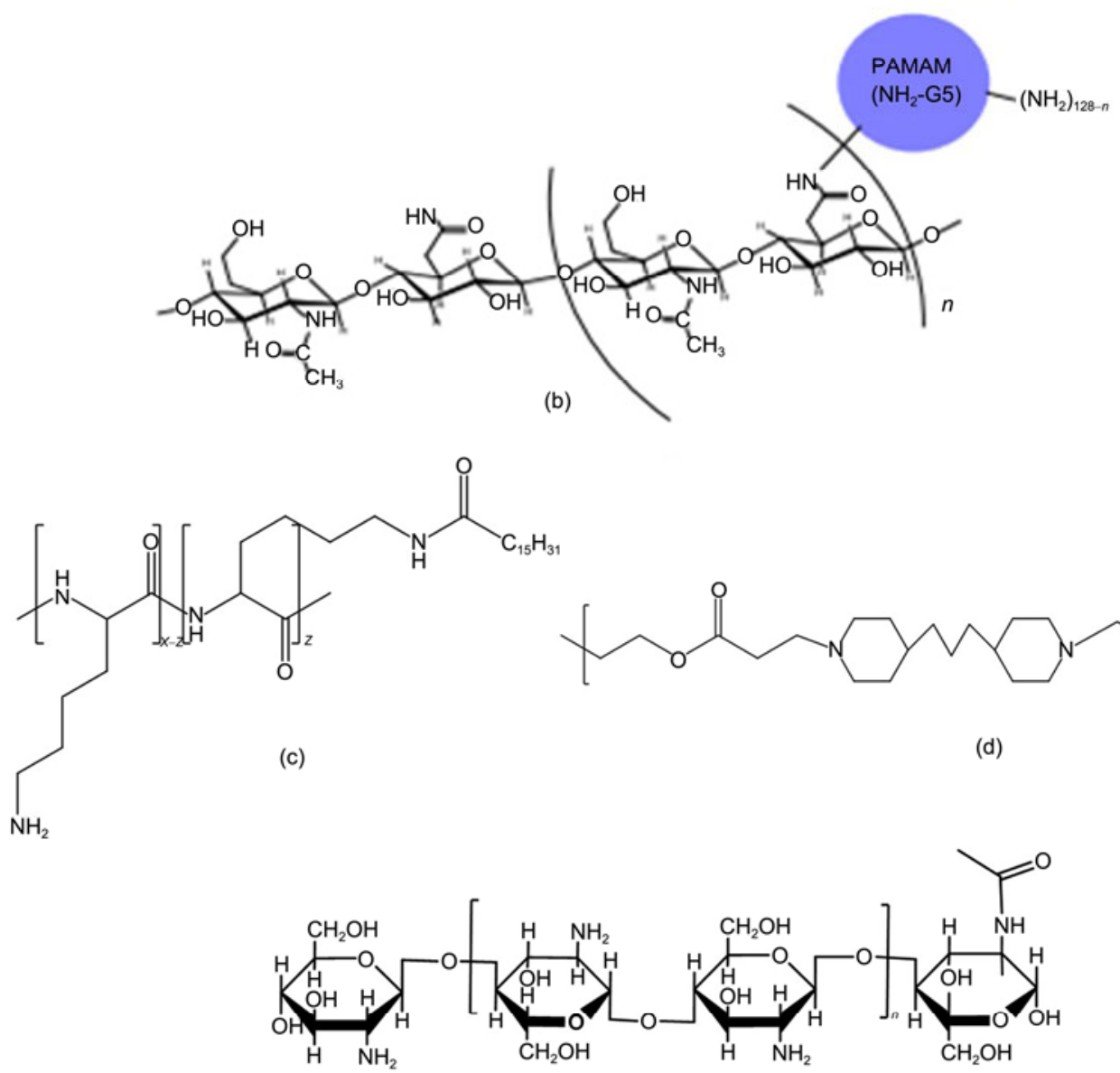

(e)

Figure 3 Cationic polymers for siRNA delivery. (a) Solid lipid PEI hybrid nanocarrier. siRNA molecules (red wavy curves) complexed with linear PEI molecules modified with hydrophobic groups (blue lines with short black branches) are physically encapsulated into nanocarrier core consisting mostly of solid lipids (pale yellow). The green surface groups represent targeting moieties (e.g. folate tagged phospholipids). Adapted with permission from [37]. Copyright (2011) American Chemical Society. (b) HA-PAMAM. Adapted with permission from [44]. Copyright (2012) Elsevier. (c) Palmitic acid-modified PLL. (d) PAE used for the preparation of PEO-modified nanoparticles. (e) Chitosan. 
et al. [39] conjugated PEI 1.8 kDa with dioleoylphosphatidylethanolamine (DOPE) for MDR1 siRNA delivery. DOPE enhanced the transfection efficacy of low-molecularweight PEI, silenced the P-gp expression, and led to a 2-fold increase of Dox uptake. Another method for optimizing PEI is employing low-molecular-weight PEI as monomers for synthesis of an entirely new cationic polymer. Liu et al. [40] synthesized a novel siRNA carrier composed of cationic PEI (1.2 kDa), a hydrophilic polymer PEG and a biodegradable lipid-based crosslinking moiety to deliver MDR1 siRNA. Reducing the expression of P-gp significantly increased the chemosensitivity of drug-resistant CD133+ cells to paclitaxel.

(2) PAMAM. Tomalia [41] originally developed PAMAM dendrimers in 1979. PAMAM dendrimers are tree-like macromolecules and have an ethylene diamine core and amido amine branches [42]. The terminated groups of half generations PAMAMs are carboxyls while the full generations are amine groups. The terminal amine groups are protonated to obtain positive charges under physiological conditions and thus the dendrimers can condense nucleic acids effectively. At the same time, the high density of surface functional groups on PAMAM dendrimers makes itself easy to be functionalized with various chemical modifications [43]. Han et al. [44] reported a polysaccharide hyaluronic acid modified PAMAM (generation 5) (HA-PAMAM) to co-deliver Dox and major vault protein (MVP) targeting siRNA (Figure 3(b)). As a result of MVP knockdown, intracelluar accumulation of Dox was increased and more Dox reached its action target, i.e. nucleus, leading to an enhanced cytotoxicity in MCF7/ADR cells.

(3) PAA. Some amino acids, such as histidine, arginine, lysine, contain basic groups in their molecular structures which can be protonated. PAAs polymerized via these amino acids have been reported to deliver siRNA and present better biocompatibility and lower toxicity than PEIs and PAMAM [45]. Poly(L-histidine) (PLH) contains imidazole groups and is uncharged at physiological conditions. So unmodified PLH can hardly be used to deliver siRNA. However, as its acid dissociation constant $\mathrm{pKa}$ is about 6.0, it will be protonated in endosomes and lysosomes, which is beneficial for endosomal escape [46]. In order to utilize this property for improved RNAi efficiency, some modified PLHs, for example, methylated PLH have been developed [47]. Arginine consists of a complex guanidinium group with a $\mathrm{pKa}$ 12.5. Poly(L-arginine) (PLA) can carry positive charges in neutral, and even basic environments. Moreover, PLA has similar characteristics as membrane-penetrating peptide and this is in favor of delivering macromolecules across the membrane $[48,49]$. Many delivery systems based on PLA have been developed to deliver siRNA [50-55]. However, the PAAs above have seldom been applied as siRNA carriers for reversing MDR. Poly(L-lysine) (PLL) is one of the most widely studied PAAs for nucleic acid delivery. It is synthesized via polymerization of $\mathrm{N}$-carboxy- anhydride of lysine [56]. PLL contains a large number of primary amino groups and could accept hydrogen ions under physiological conditions. Lipid modification of PLL are beneficial for the stability of loaded siRNA and the interaction between delivery system and cell membranes, which contribute to enhanced transport of loaded siRNA or DNA. Abbasi et al. [57] employed palmitic-acid substituted poly (L-lysine) as carrier for MDR1 siRNA (Figure 3(c)). Efficient siRNA delivery was observed in both wild type and $\mathrm{P}$-gp-overexpressing phenotype cell lines. It resulted in approximately $40 \%$ to $50 \%$ P-gp suppression (based on the average expression level of the protein) and an approximately 3-fold increased Dox uptake. It increased cytotoxicity of both Dox and paclitaxel in P-gp-overexpressing MDA435/LCC6 cells.

(4) PAE. PAE is a kind of cationic polymers synthesized via Michael addition reactions between compounds containing primary amine or secondary amine and diacrylates and is a promising non-viral gene delivery system. PAE is able to condense DNA or siRNA into stable nanoparticles at physiological $\mathrm{pH}$. Hydrolytic cleavable ester groups in its chemical structure make PAE biodegradable and show lower cytotoxicity when compared with some other cationic polymers. An ideal PAE can be designed by means of changing the monomer of polymer. Zugates et al. [58-61] established a library of PAEs which have diverse compositions and structures and characterized them through high-throughput screening methods. Our laboratory [62,63] synthesized a series of bioreducible PAEs, which contains disulfide bonds in its structure. Bioreducible PAEs could response to the tumor cells' intracellular reducing environment and realize prompt RNA release. Poly (ethylene oxide) (PEO)-modified PAE nanoparticles were prepared by Yadav et al. [64] to efficiently encapsulate MDR1 siRNA (Figure 3(d)). PEO-modified poly(epsilon-caprolactone) (PEO-PCL) nanoparticles loaded with paclitaxel were also prepared. The siRNA complexes could down-regulate the P-gp expression in multidrug resistant SKOV3TR human ovarian adenocarcinoma cells, contributing to increased intracellular paclitaxel accumulation and enhanced the cytotoxicity of paclitaxel nanoparticles to SKOV3TR cells.

(5) Chitosan. Chitosan, a deacetylation derivative of chitin, is a natural linear alkaline polysaccharide (Figure 3(e)). It is a particularly attractive cationic polymer for biomedical applications due to its good biocompatibility, biodegradability, negligible immunogenicity, and low toxicity $[65,66]$. The primary amine groups in repeated glucosamine unit of chitosan can be protonated under acidic conditions. Therefore chitosan can be utilized as siRNA carriers for RNAi. Ziebarth et al. [67] deliver endoglin (CD105) siRNA by its chitosan complexes in MDR ES2 and HeyA8 mouse models. Chitosan-encapsulated siRNA promoted the apoptosis of tumor cells and improves their sensitivity against platinum. The in vivo experiment results showed that tumor weights of endoglin siRNA treatment group were reduced 
by $35 \%-41 \%$ compared with control and the endoglin siRNA combined with carboplatin group were even better $(58 \%-$ $62 \%$ ). Steg et al. [68] used chitosan nanoparticles to deliver Jagged1 siRNA in IGROV-AF1 and taxane-resistant SKOV3TRip2 mouse models. The complexes efficiently down-regulated the expression of Jagged1 and sensitized SKOV3TRip2 tumors to docetaxel. Combination of Jagged1 siRNA complexes and docetaxel reduced tumor weight by $87.5 \%-93.1 \%$. Microvessel density in tumor was reduced and the proliferation of tumor cells was inhibited. The author pointed that Jagged1 down-regulation might sensitize tumor cells to taxanes by cross-talk with the GLI2 mediator of the Hedgehog pathway rather than through reduction in MDR1 expression. In Jagani et al.'s [69] work, chitosan nanoparticles were prepared via ionic gelation of chitosan by tripolyphosphate for delivery of Bcl2 siRNA in HeLa cell and mouse models. Blocking the expression of $\mathrm{Bcl} 2$ gene by the chitosan nanoparticles increased the apoptosis rate of tumor cells, enhanced the chemosensitivity to cisplatin and significantly reduced the tumor volumes in vivo.

(6) Cationic polymers synthesized by Reversible Addition-Fragmentation Chain Transfer (RAFT) Polymerization. RAFT polymerization, which was first reported by the Australian CSIRO group, is particularly promising as a means for designing and synthesizing novel polymers as nuclear acid drug vectors [70,71]. It is a robust method by which one can control the molecular weight, composition, architecture and functionality of the polymers. RAFT polymerization does not need metal catalysts and can be done in various ranges of reaction conditions [72]. Some monomers without reactive groups (such as hydroxyl groups, carboxyl groups, amino groups) can also be polymerized via this method. RAFT process contains a chain transfer agent with a thiocarbonylthio group, which participates in the following formation of intermediate radical species and is an important factor for successful polymerization [73,74]. Cationic polymers synthesized by RAFT have been reported as siRNA carriers to overcome MDR in cancer. Benoit et al. [75] prepared a cationic micelle formed from diblock copolymers of dimethylaminoethyl methacrylate (pDMAEMA) and butyl methacrylate. pDMAEMA contained tertiary amine groups and carried positive charges under physiological conditions. It endowed the micelles with the ability to deliver polo-like Kinase 1 (plk1) siRNA. In order to enhance cytosolic delivery through endosomal release, $\mathrm{pH}$-responsive copolymer of poly(styrene-alt-maleic anhydride) was electrostatically complexed with the positively charged siRNA/ micelle to form a ternary complex. Treatment with the ternary complexes reduced plk1 gene expression by $50 \%$ in both the drug-resistant NCI/ADR-RES cell line and the drugsensitive OVCAR8 cell line. It resulted in the sensitization of NCI/ADR-RES cells to doxorubicin. The author demonstrated that this phenomenon is related to p53 signaling pathway. Micelles co-delivering plk1 siRNA and Dox resulted in a synergistic effect and sensitize MDR cells to Dox.

\subsection{Inorganic carriers}

Inorganic nanoparticles are attracting more and more interest as siRNA or drug carriers because they are easy to be prepared with a defined and stable structures and sizes. Various kinds of inorganic materials, such as porous silica nanoparticles, gold nanoparticles, nanorods and carbonate apatite nanoparticles, have been investigated for delivering genes or theraputic agents.

(1) Porous silicon nanoparticles. Porous silicon nanoparticles have been considered as excellent candidates for medical applications due to their remarkably large surface area and biocompatibility [76-78]. They can be endowed with more functions via decorating them with various kinds of organic polymers. Meng et al. [79] prepared mesoporous silicon nanoparticles functionalized by phosphonate groups, which allowed electrostatic binding of Dox to the porous interior (Figure 4(a)). With the help of phosphonate groups, the nanoparticle was then coated with branched PEI. The two modifications made it possible to co-deliver Dox and MDR1 siRNA to drug-resistant cancer cell line KB-V1 cells. The co-delivery system increased the intracelluar and intranuclear Dox concentration to levels exceeding that of free Dox or the mesoporous silicon nanoparticles loading Dox alone and it improved the sensitivity of the drug-resistant cells to Dox. Recently, the same group [80] prepared a PEIPEG copolymer functionalized mesoporous silica nanoparticle to overcome Dox resistance in a multidrug resistant human breast cancer xenograft by co-delivering Dox and MDR1 siRNA. The siRNA was selected by high throughput screening method in MCF7/MDR cells. The co-delivery system resulted in stronger inhibition of tumor growth in a MDR tumor xenograft model in vivo than free Dox or the nanoparticles loaded with either drug or siRNA alone. The researchers also analyzed the impact of heterogeneity in the tumor microenvironment on the efficacy of siRNA delivery in vivo and proposed that the delivery system could be further improved by addressing intratumor uneven distribution. Mesoporous silicon nanoparticles modified with PAMAM dendrimers (generation 2) was investigated by Chen et al. [81] for co-delivering Dox and Bcl2 siRNA into multidrug resistant cancer cells. Silicon nanoparticles firstly reacted with 3-iso-cyanatopropyltriethoxysilane to yield isocyanatopropyl-modified surface and then were linked to PAMAM dendrimers (generation 2). This delivery system increased the anticancer efficacy of Dox by 132 times in drug resistant A2780/AD ovarian cancer cells compared to free Dox because of the synergistic effect of the Dox and Bcl2 siRNA.

(2) Gold nanoparticles (AuNPs). AuNPs have been widely investigated for their good bioinertia, lower toxicity, ready synthesis, and easy functionalization [82]. Han et al. [83] constructed a $\mathrm{pH}$-sensitive gold nanoparicle coated with chitosan, PEI and PAH-Cit as a gene vehicle using a layer-by-layer assembly method (Figure 4(b)). First, the AuNPs were stabilized by chitosan, forming a positively 


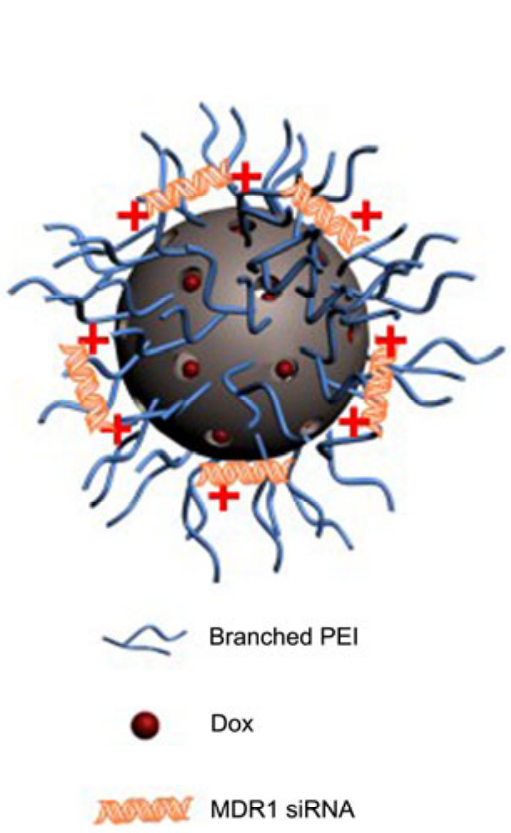

(a)

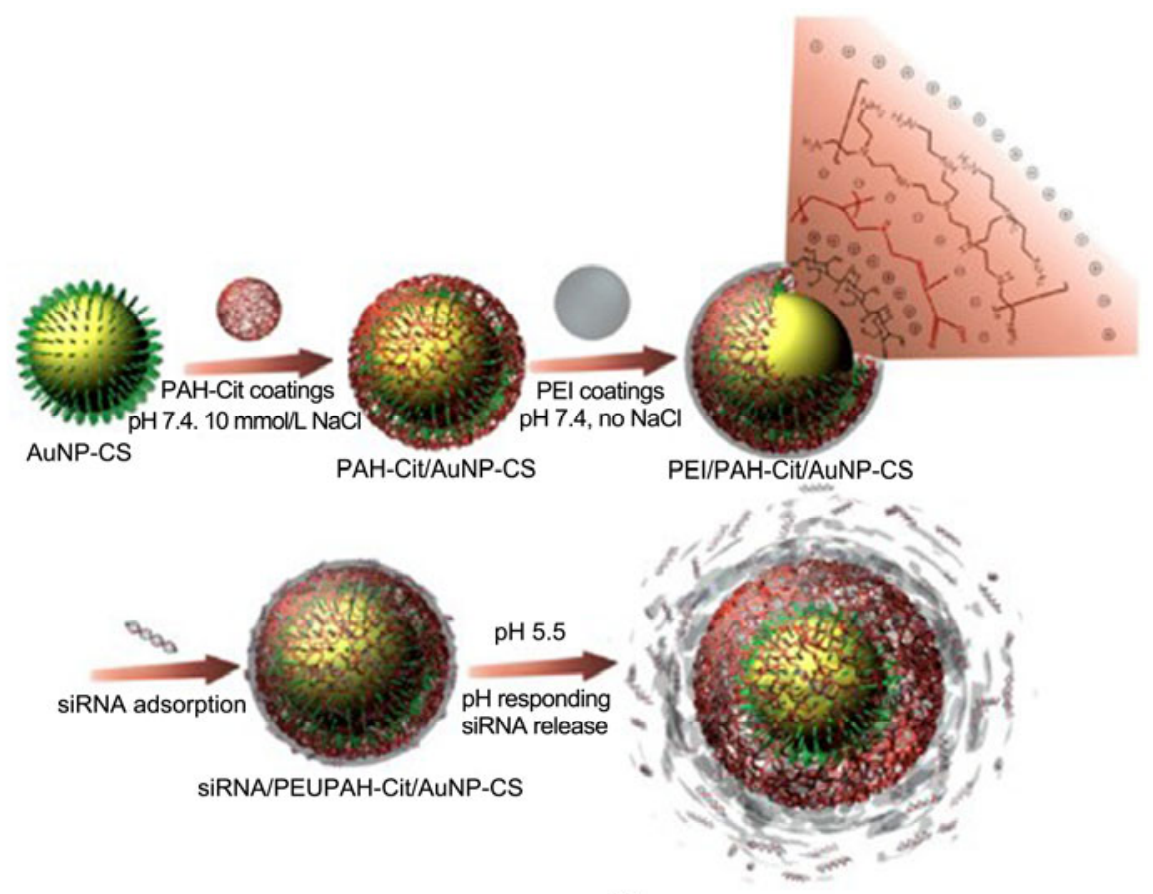

(b)

Figure 4 Inorganic nanocarriers for siRNA delivery. (a) Mesoporous silicon nanoparticle modified with branched PEI. (b) Assembly steps for siRNA/ PEI/PAH-Cit/AuNP-CS complexes and pH-responsive release of siRNA. Adapted with permission from [78]. Copyright (2012) American Chemical Society.

charged AuNP-CS core. Then the charge-reversible PAHCit and PEI were deposited onto the surface of AuNP-CS through electrostatic interaction. PEI/PAH-Cit/AuNP-CS loaded with siRNA showed $79 \%$ siRNA release in $4 \mathrm{~h}$ at $\mathrm{pH}$ 5.5 in vitro, suggesting a $\mathrm{pH}$-induced charge-reversing action of PAH-Cit. This mechanism also worked in vivo and facilitated the escape of siRNA from endosomes. The author used P-gp targeted siRNA/PEI/PAH-Cit/AuNP-CS to knockdown the expression of MDR1 gene in drug-resistant MCF7 cells and consequently increased uptake of Dox was observed.

(3) Apatite nanoparticles. Apatite is a general term for phosphate minerals, mainly referring to fluorapatite, hydroxylapatite, carbonate apatite, and chlorapatite. It has been used as materials for orthopedic and dental applications because of their resemblance to body hard tissue components and good biodegradability and biocompatibility $[84,85]$. Apatite nanoparticles can also serve as efficient nuclear acid drugs carriers for RNAi. siRNA or shRNA could be loaded in apatite nanoparticles by the method of calcium-phosphate coprecipitation. In the process of coprecipitation, divalent calcium ions can form ionic complexes with the phosphates of nuclear acid. Then the siRNA or shRNA was immobilized in calcium phosphate layers and was protected from external damage before reaching the target site [86]. Li et al. [87] developed $\mathrm{pH}$-sensitive carbonate apatite nanoparticles, which were able to co-deliver two kinds of siRNA targeting MDR1 and ABCG2 to MCF7 cells. Carbonate apatite nanoparticles showed high affinity interactions with siRNA, facilitating transportation of siRNA across the cell membrane. Delivery of single siRNA targeting either ABCG2 or MDR1 transporter with carbonate apatite nanoparticles demonstrated only about $20 \%$ enhancement in sensitivity to cisplatin, paclitaxel, or Dox in MCF7 cells, while co-delivery of siRNA targeting MDR1 and ABCG2 to MCF7 cells increased the chemosensitivity by about $47 \%$, indicating the reversal of $\mathrm{ABC}$ transportermediated multidrug resistance.

\subsection{Other nanocarriers}

Some polymers, such as $\beta$-cyclodextrin, PEO-block-poly ( $\varepsilon$ caprolactone) (PCL) and dextran, which do not contain amino groups in their chemical structures, can also deliver siRNA after appropriate modification [88-92]. Li et al. [88] developed a MDR1 siRNA and Dox co-delivery system, in which $\mathrm{CdSe} / \mathrm{ZnSe}$ quantum dots were coated with $\beta$-cyclodextrin via amino acids L-Arg or L-His. The $\beta$-cyclodextrin was used to encapsulate Dox and the linkers L-Arg or L-His bound with siRNA to form stable complexes. The delivery system co-localized MDR1 siRNA and Dox in drug resistant HeLa/Dox cells and generated synergistic effects of the chemotherapeutic and gene therapy agents, leading to reduced levels of MDR1 gene expression, enhanced intracellular Dox accumulation and an increased potency of Dox to induce apoptosis in HeLa/Dox cells. PCL is one kind of the FDA-approved biomaterials. The PCL derivative with reactive carboxyl groups, poly ( $\alpha$-carboxyl- $\varepsilon$-caprolactone) 
(PCCL) can be obtained by replacement of the monomer $\varepsilon$-caprolactone with $\alpha$-benzyl carboxylate- $\varepsilon$-caprolactone in PCL polymerization. With the help of $\alpha$-carboxyl groups, one can endow the PCL polymer with more functionality, including the abilities to deliver nuclear acid drug. Xiong et al. [89,90] modified PEO-PCCL with spermine or $N, N$ dimethyldipropylenetriamine. Amino groups introduced in its structures bound siRNA stably via electrostatic attraction under physiological conditions. The PEO blocks were able to be decorated with integrin $\alpha v \beta 3$ targeting peptide (RGD4C) and/or cell penetrating peptide (TAT), increasing the specificity to MDR cancer cells. Dox was further conjugated to PEO-PCCL via $\mathrm{pH}$-sensitive hydrazone bond forming a codelivery system of Dox and MDR1 siRNA [90]. This system significantly increased cellular uptake, improved Dox penetration into nuclei, and finally enhanced Dox cytotoxicity in Dox-resistant MDA-MB-435 cancer models. Dextran can be modified with various kinds of functional groups easily due to its plentiful reactive hydroxyl groups. A lipidmodified dextran-based polymeric nanoparticles, containing dextran thiol, dextran stearylamine, and PEG-thiol were reported to deliver MDR1 siRNA by Susa et al. [91]. The siRNA complexes could efficiently suppress P-gp expression in the drug resistant osteosarcoma cell lines (KHOSR2 and U-2OSR2) and re-sensitize these cancer cells to Dox. A similar lipid-modified dextran nanosystem, in which dextran stearylamine was replaced by dextran octylamine, was also assessed to deliver MDR1 siRNA by their group [92].

Nanogels, which have been widely used in drug delivery and medical diagnosis, can also deliver siRNA to targeted cells [93]. Nanogels are able to be loaded with siRNA via "breathing-in" manner besides electrostatic attraction [94]. Dickerson et al. [95] investigated core/shell nanogels composed of poly ( $N$-isopropylmethacrylamide) (pNIPMAm) cross-linked with $N, N^{\prime}$-methylene(bisacrylamide) to deliver siRNA targeting epidermal growth factor receptor (EGFR). Knockdown of (EGFR) contributes to the overcoming of MDR in cancer cells [96]. The surface of the nanogels were functionalized with peptides (YSA) that specially target erythropoietin-producing hepatocellular (Eph)A2 receptor, which is up-regulated in vasculature of human tumors. The SDS decreased EGFR expression in Hey cells, with the highest RNAi efficiency occurring at $48 \mathrm{~h}$ and significantly increased the sensitivity of this cell line to docetaxel. It is worth noting that the specificity of this delivery system was confirmed by the experiment of failed reduction of EGFR levels in (Eph)A2 negative SK-OV-3 cells.

\section{Conclusion and future direction}

Various kinds of nanocarriers have been developed for delivering siRNA in order to overcome tumor MDR in recent years. ABC transporters were chosen as the targets in most studies. However, mechanisms involved in MDR in tumors are very complicated. The generation of MDR phenotype is usually a collective result of several mechanisms. Designing a nanocarrier that can co-deliver several kinds of siRNA against different mechanisms may be one of the solutions to overcome MDR. More and more knowledge of cancer biology and MDR is revealed and more opportunities are opened up for reversing MDR. At the same time, with the development of material sciences, especially biodegradable polymer materials, more well-designed and complicated nanocarriers can be synthesized, which increased the possibility of successful treatment to cancer.

It is worth noting that there have been few siRNA complexes for systemic administration entering clinical trials [97]. One of the solutions to improve this situation is increasing the specificity of siRNA nanocarriers to targeted tumor cells and decreasing cytotoxicity to normal cells by active targeting or passive targeting. Moreover, the variations of the physicochemical and biological properties of the SDS during their in vivo process still need exploring. And the approaches to prepare siRNA complexes should be optimized for quality assurance of products.

This work was supported by the National Basic Research Program of China (2010CB934000, 2012CB932502, 2013CB932503), the National Natural Science Foundation of China (30925041, 81270047) and Shanghai Elitist Program (11XD1406200).

1 Harris A L, Hochhauser D. Mechanisms of multidrug resistance in cancer treatment. Acta Oncol, 1992, 31: 205-213

2 Gottesman M M, Fojo T, Bates S E. Multidrug resistance in cancer: Role of ATP-dependent transporters. Nat Rev Cancer, 2002, 2: 48-58

3 Szakacs G, Paterson J K, Ludwig J A, et al. Targeting multidrug resistance in cancer. Nat Rev Drug Discov, 2006, 5: 219-234

4 Gillet J P, Gottesman M M. Mechanisms of multidrug resistance in cancer. Methods Mol Biol, 2010, 596: 47-76

5 Elbashir S M, Harborth J, Lendeckel W, et al. Duplexes of 21-nucleotide RNAs mediate RNA interference in cultured mammalian cells. Nature, 2001, 411: 494-498

6 Kawasaki H, Taira K, Morris K V. siRNA induced transcriptional gene silencing in mammalian cells. Cell Cycle, 2005, 4: 442-448

7 Schutze N. siRNA technology. Mol Cell Endocrinol, 2004, 213: 115-119

8 Shim M S, Kwon Y J. Efficient and targeted delivery of siRNA in vivo. FEBS J, 2010, 277: 4814-4827

9 Chen Y, Huang L. Tumor-targeted delivery of siRNA by non-viral vector: Safe and effective cancer therapy. Expert Opin Drug Deliv, 2008, 5: 1301-1311

10 Huang X, Yang Y. Innate immune recognition of viruses and viral vectors. Hum Gene Ther, 2009, 20: 293-301

11 Aigner A. Applications of RNA interference: Current state and prospects for siRNA-based strategies in vivo. Appl Microbiol Biotechnol, 2007, 76: 9-21

12 Ozpolat B, Sood A K, Lopez-Berestein G. Nanomedicine based approaches for the delivery of siRNA in cancer. J Intern Med, 2010, 267: 44-53

13 Tong A W, Jay C M, Senzer N, et al. Systemic therapeutic gene delivery for cancer: Crafting Paris' arrow. Curr Gene Ther, 2009, 9: 45-60

14 Zelphati O, Uyechi L S, Barron L G, et al. Effect of serum components on the physico-chemical properties of cationic lipid/oligonucleotide complexes and on their interactions with cells. Biochim Biophys Acta, 1998, 1390: 119-133 
15 Khalil I A, Kogure K, Akita H, et al. Uptake pathways and subsequent intracellular trafficking in nonviral gene delivery. Pharmacol Rev, 2006, 58: 32-45

16 Rejman J, Oberle V, Zuhorn I S, et al. Size-dependent internalization of particles via the pathways of clathrin- and caveolae-mediated endocytosis. Biochem J, 2004, 377: 159-169

17 Sato A, Takagi M, Shimamoto A, et al. Small interfering RNA delivery to the liver by intravenous administration of galactosylated cationic liposomes in mice. Biomaterials, 2007, 28: 1434-1442

18 Schiffelers R M, Ansari A, Xu J, et al. Cancer siRNA therapy by tumor selective delivery with ligand-targeted sterically stabilized nanoparticle. Nucleic Acids Res, 2004, 32: e149

19 Pirollo K F, Rait A, Zhou Q, et al. Materializing the potential of small interfering RNA via a tumor-targeting nanodelivery system. Cancer Res, 2007, 67: 2938-2943

20 Song E, Zhu P, Lee S K, et al. Antibody mediated in vivo delivery of small interfering RNAs via cell-surface receptors. Nat Biotechnol, 2005, 23: 709-717

21 Varkouhi A K, Scholte M, Storm G, et al. Endosomal escape pathways for delivery of biologicals. J Control Release, 2011, 151: 220228

22 Bhavsar D, Subramanian K, Sethuraman S, et al. Translational siRNA therapeutics using liposomal carriers: Prospects \& challenges. Curr Gene Ther, 2012, 12: 315-332

23 Saad M, Garbuzenko O B, Minko T. Co-delivery of siRNA and an anticancer drug for treatment of multidrug-resistant cancer. Nanomedicine, 2008, 3: 761-776

24 Chen Y, Bathula S R, Li J, et al. Multifunctional nanoparticles delivering small interfering RNA and doxorubicin overcome drug resistance in cancer. J Biol Chem, 2010, 285: 22639-22650

25 Nakamura K, Abu Lila A S, Matsunaga M, et al. A double-modulation strategy in cancer treatment with a chemotherapeutic agent and siRNA. Mol Ther, 2011, 19: 2040-2047

26 Jiang J, Yang S J, Wang J C, et al. Sequential treatment of drugresistant tumors with RGD-modified liposomes containing siRNA or doxorubicin. Eur J Pharm Biopharm, 2010, 76: 170-178

27 Halder J, Kamat A A, Landen C N Jr, et al. Focal adhesion kinase targeting using in vivo short rfering RNA delivery in neutral liposomes for ovarian carcinoma therapy. Clin Cancer Res, 2006, 12: 4916-4924

28 Samal S K, Dash M, Van Vlierberghe S, et al. Cationic polymers and their therapeutic potential. Chem Soc Rev, 2012, 41: 7147-7194

29 Jeong J H, Kim S W, Park T G. Molecular design of functional polymers for gene therapy. Prog Polym Sci, 2007, 32: 1239-1274

30 Grayson A C, Doody A M, Putnam D. Biophysical and structural characterization of polyethylenimine-mediated siRNA delivery in vitro. Pharm Res, 2006, 23: 1868-1876

31 Oskuee R K, Philipp A, Dehshahri A, et al. The impact of carboxyalkylation of branched polyethylenimine on effectiveness in small interfering RNA delivery. J Gene Med, 2010, 12: 729-738

32 Jere D, Jiang H L, Arote R, et al. Degradable polyethylenimines as DNA and small interfering RNA carriers. Expert Opin Drug Deliv, 2009, 6: 827-834

33 Neu M, Fischer D, Kissel T. Recent advances in rational gene transfer vector design based on poly(ethylene imine) and its derivatives. J Gene Med, 2005, 7: 992-1009

34 Hunter A C. Molecular hurdles in polyfectin design and mechanistic background to polycation induced cytotoxicity. Adv Drug Deliv Rev, 2006, 58: 1523-1531

35 Breunig M, Lungwitz U, Liebl R, et al. Breaking up the correlation between efficacy and toxicity for nonviral gene delivery. Proc Natl Acad Sci USA, 2007, 104: 14454-14459

36 Aliabadi H M, Landry B, Mahdipoor P, et al. Effective down-regulation of breast cancer resistance protein (BCRP) by siRNA delivery using lipid-substituted aliphatic polymers. Eur J Pharm Biopharm, 2012, 81: 33-42

37 Xue H Y, Wong H L. Solid lipid-PEI hybrid nanocarrier: An integrated approach to provide extended, targeted, and safer siRNA therapy of prostate cancer in an all-in-one manner. ACS Nano, 2011, 5:
7034-7047

38 Xue H Y, Wong H L. Targeting megalin to enhance delivery of anticlusterin small-interfering RNA nanomedicine to chemo-treated breast cancer. Eur J Pharm Biopharm, 2012, 81: 24-32

39 Navarro G, Sawant R R, Biswas S, et al. P-glycoprotein silencing with siRNA delivered by DOPE-modified PEI overcomes doxorubicin resistance in breast cancer cells. Nanomedicine (Lond), 2012, 7: 65-78

40 Liu C, Zhao G, Liu J, et al. Novel biodegradable lipid nano complex for siRNA delivery significantly improving the chemosensitivity of human colon cancer stem cells to paclitaxel. J Control Release, 2009, 140: 277-283

41 Tomalia D A, Baker H, Dewald J, et al. A new class of polymers: Starburst-dendritic macromolecules. Polymer, 1985, 17: 117-132

42 Sadekar S, Ghandehari H. Transepithelial transport and toxicity of PAMAM dendrimers: Implications for oral drug delivery. Adv Drug Deliv Rev, 2012, 64: 571-588

43 Pettit M W, Griffiths P, Ferruti P, et al. Poly(amidoamine) polymers: Soluble linear amphiphilic drug-delivery systems for genes, proteins and oligonucleotides. Ther Deliv, 2011, 2: 907-917

44 Han M, Lv Q, Tang X J, et al. Overcoming drug resistance of MCF7/ADR cells by altering intracellular distribution of doxorubicin via MVP knockdown with a novel siRNA polyamidoamine-hyaluronic acid complex. J Control Release, 2012, 163: 136-144

45 Vorhies J S, Nemunaitis J J. Synthetic vs. Natural/biodegradable polymers for delivery of shRNA-based cancer therapies. Methods Mol Biol, 2009, 480: 11-29

46 Midoux P, Kichler A, Boutin V, et al. Membrane permeabilization and efficient gene transfer by a peptide containing several histidines. Bioconjug Chem, 1998, 9: 260-267

47 Asayama S, Kumagai T, Kawakami H. Synthesis and characterization of methylated poly(L-histidine) to control the stability of its siRNA polyion complexes for RNAi. Bioconjug Chem, 2012, 23: 1437-1442

48 Mitchell D J, Kim D T, Steinman L, et al. Polyarginine enters cells more efficiently than other polycationic homopolymers. J Pept Res, 2000, 56: 318-325

49 Wender P A, Mitchell D J, Pattabiraman K, et al. The design, synthesis, and evaluation of molecules that enable or enhance cellular uptake: Peptoid molecular transporters. Proc Natl Acad Sci USA, 2000, 97: 13003-13008

50 Kim E J, Shim G, Kim K, et al. Hyaluronic acid complexed to biodegradable poly L-arginine for targeted delivery of siRNAs. J Gene Med, 2009, 11: 791-803

51 Cho H J, Chong S, Chung S J, et al. Poly-L-arginine and dextran sulfate-based nanocomplex for epidermal growth factor receptor (EGFR) siRNA delivery: Its application for head and neck cancer treatment. Pharm Res, 2012, 29: 1007-1019

52 Won Y W, Yoon S M, Lee K M, et al. Poly(oligo-D-arginine) with internal disulfide linkages as a cytoplasm-sensitive carrier for siRNA delivery. Mol Ther, 2011, 19: 372-380

53 Kim H K, Davaa E, Myung C S, et al. Enhanced siRNA delivery using cationic liposomes with new polyarginine-conjugated PEG-lipid. Int J Pharm, 2010, 392: 141-147

54 Noh S M, Park M O, Shim G, et al. Pegylated poly-L-arginine derivatives of chitosan for effective delivery of siRNA. J Control Release, 2010, 145: 159-164

55 Kim S H, Jeong J H, Kim T I, et al. VEGF siRNA delivery system using arginine-grafted bioreducible poly(disulfide amine). Mol Pharm, 2009, 6: 718-726

56 Zhang S, Xu Y, Wang B, et al. Cationic compounds used in lipoplexes and polyplexes for gene delivery. J Control Release, 2004, 100: 165180

57 Abbasi M, Lavasanifar A, Berthiaume L G, et al. Cationic polymermediated small interfering RNA delivery for P-glycoprotein downregulation in tumor cells. Cancer, 2010, 116: 5544-5554

58 Zugates G T, Tedford N C, Zumbuehl A, et al. Gene delivery properties of end-modified poly(beta-amino ester)s. Bioconjug Chem, 2007, 18: $1887-1896$ 
59 Zugates G T, Peng W, Zumbuehl A, et al. Rapid optimization of gene delivery by parallel end-modification of poly(beta-amino ester)s. Mol Ther, 2007, 15: 1306-1312

60 Sunshine J C, Peng D Y, Green J J. Uptake and transfection with polymeric nanoparticles are dependent on polymer end-group structure, but largely independent of nanoparticle physical and chemical properties. Mol Pharm, 2012, 9: 3375-3383

61 Anderson D G, Akinc A, Hossain N, et al. Structure/property studies of polymeric gene delivery using a library of poly(beta-amino esters). Mol Ther, 2005, 11: 426-434

62 Yin Q, Gao Y, Zhang Z, et al. Bioreducible poly (beta-amino esters)/ shRNA complex nanoparticles for efficient RNA delivery. J Control Release, 2011, 151: 35-44

63 Yin Q, Shen J, Chen L, et al. Overcoming multidrug resistance by codelivery of Mdr-1 and survivin-targeting RNA with reduction-responsible cationic poly( $\beta$-amino esters). Biomaterials, 2012, 33: 64956506

64 Yadav S, van Vlerken L E, Little S R, et al. Evaluations of combination MDR-1 gene silencing and paclitaxel administration in biodegradable polymeric nanoparticle formulations to overcome multidrug resistance in cancer cells. Cancer Chemoth Pharm, 2009, 63: 711722

65 Han H D, Song C K, Park Y S, et al. A chitosan hydrogel-based cancer drug delivery system exhibits synergistic antitumor effects by combining with a vaccinia viral vaccine. Int J Pharm, 2008, 350: 27-34

66 Seo S H, Han H D, Noh K H, et al. Chitosan hydrogel containing GMCSF and a cancer drug exerts synergistic anti-tumor effects via the induction of CD8+ t cell-mediated anti-tumor immunity. Clin Exp Metastasis, 2009, 26: 179-187

67 Ziebarth A J, Nowsheen S, Steg A D, et al. Endoglin (CD105) contributes to platinum resistance and is a target for tumor-specific therapy in epithelial ovarian cancer. Clin Cancer Res, 2013, 19: 170-182

68 Steg A D, Katre A A, Goodman B, et al. Targeting the notch ligand JAGGED1 in both tumor cells and stroma in ovarian cancer. Clin Cancer Res, 2011, 17: 5674-5685

69 Jagani H, Rao J V, Palanimuthu V R, et al. A nanoformulation of siRNA and its role in cancer therapy: In vitro and in vivo evaluation. Cell Mol Biol Lett, 2013, 18: 120-136

70 Chiefari J, Chong Y K, Ercole F, et al. Living free-radical polymerization by reversible addition-fragmentation chain transfer: The RAFT process. Macromolecules, 1998, 31: 5559-5562

71 Moad G, Rizzardo E, Thang S H. Living radical polymerization by the RAFT process - a first update. Aust J Chem, 2006, 59: 669-692

72 Fallahi H, Koohmareh G A. Preparation of polystyrene/MMT nanocomposite through in situ RAFT polymerization by new chain transfer agent derived from bisphenol A. J Appl Polym Sci, 2013, 127: 523-529

73 Gregory A, Stenzel M H. Complex polymer architectures via RAFT polymerization: From fundamental process to extending the scope using click chemistry and nature's building blocks. Prog Polym Sci, 2012, 37: 38-105

$74 \mathrm{Xu}$ F J, Yang W T. Polymer vectors via controlled/living radical polymerization for gene delivery. Prog Polym Sci, 2011, 36: 10991131

75 Benoit D S, Henry S M, Shubin A D, et al. pH-responsive polymeric siRNA carriers sensitize multidrug resistant ovarian cancer cells to doxorubicin via knockdown of polo-like kinase 1. Mol Pharm, 2010, 7: 442-455

76 Yanes R E, Tamanoi F. Development of mesoporous silica nanomaterials as a vehicle for anticancer drug delivery. Ther Deliv, 2012, 3: $389-404$

77 Godin B, Tasciotti E, Liu X, et al. Multistage nanovectors: From concept to novel imaging contrast agents and therapeutics. Acc Chem Res, 2011, 44: 979-989
78 Buriak J M. High surface area silicon materials: Fundamentals and new technology. Philos Transact A Math Phys Eng Sci, 2006, 364: 217-225

79 Meng H, Liong M, Xia T, et al. Engineered design of mesoporous silica nanoparticles to deliver doxorubicin and P-glycoprotein siRNA to overcome drug resistance in a cancer cell line. ACS Nano, 2010, 4: 4539-4550

80 Meng H, Mai W X, Zhang H, et al. Codelivery of an optimal drug/ siRNA combination using mesoporous silica nanoparticles to overcome drug resistance in breast cancer in vitro and in vivo. ACS Nano, 2013, 7: 994-1005

81 Chen A M, Zhang M, Wei D, et al. Co-delivery of doxorubicin and Bcl-2 siRNA by mesoporous silica nanoparticles enhances the efficacy of chemotherapy in multidrug-resistant cancer cells. Small, 2009, 5: 2673-2677

82 Lytton-Jean A K, Langer R, Anderson D G. Five years of siRNA delivery: Spotlight on gold nanoparticles. Small, 2011, 7: 1932-1937

83 Han L, Zhao J, Zhang X, et al. Enhanced siRNA delivery and silencing gold-chitosan nanosystem with surface charge-reversal polymer assembly and good biocompatibility. ACS Nano, 2012, 6: 7340-7351

84 Chowdhury E H, Maruyama A, Kano A, et al. pH-sensing nanocrystals of carbonate apatite: Effects on intracellular delivery and release of DNA for efficient expression into mammalian cells. Gene, 2006, 376: 87-94

85 Chowdhury E H, Akaike T. A bio-recognition device developed onto nano-crystals of carbonate apatite for cell-targeted gene delivery. Biotechnol Bioeng, 2005, 90: 414-421

86 Roy I, Mitra S, Maitra A, et al. Calcium phosphate nanoparticles as novel non-viral vectors for targeted gene delivery. Int J Pharm, 2003, 250: 25-33

87 Li Y T, Chua M J, Kunnath A P, et al. Reversing multidrug resistance in breast cancer cells by silencing $\mathrm{ABC}$ transporter genes with nanoparticle-facilitated delivery of target siRNAs. Int J Nanomedicine, 2012, 7: 2473-2481

88 Li J M, Wang Y Y, Zhao M X, et al. Multifunctional QD-based co-delivery of siRNA and doxorubicin to HeLa cells for reversal of multidrug resistance and real-time tracking. Biomaterials, 2012, 33: 2780-2790

89 Xiong X B, Uludag H, Lavasanifar A. Virus-mimetic polymeric micelles for targeted siRNA delivery. Biomaterials, 2010, 31: 58865893

90 Xiong X B, Lavasanifar A. Traceable multifunctional micellar nanocarriers for cancer-targeted co-delivery of MDR-1 siRNA and doxorubicin. ACS Nano, 2011, 5: 5202-5213

91 Susa M, Iyer A K, Ryu K, et al. Inhibition of ABCB1 (MDR1) expression by an siRNA nanoparticulate delivery system to overcome drug resistance in osteosarcoma. PLoS One, 2010, 5: e10764

92 Kobayashi E, Iyer A K, Hornicek F J, et al. Lipid-functionalized dextran nanosystems to overcome multidrug resistance in cancer: A pilot study. Clin Orthop Relat Res, 2012

93 Smith M H, Lyon L A. Multifunctional nanogels for siRNA delivery. Acc Chem Res, 2012, 45: 985-993

94 Blackburn W H, Dickerson E B, Smith M H, et al. Peptide-functionalized nanogels for targeted siRNA delivery. Bioconjug Chem, 2009, 20: 960-968

95 Dickerson E B, Blackburn W H, Smith M H, et al. Chemosensitization of cancer cells by siRNA using targeted nanogel delivery. BMC Cancer, 2010, 10: 10

96 Thaker P H, Yazici S, Nilsson M B, et al. Antivascular therapy for orthotopic human ovarian carcinoma through blockade of the vascular endothelial growth factor and epidermal growth factor receptors. Clin Cancer Res, 2005, 11: 4923-4933

97 David S, Pitard B, Benoit J P, et al. Non-viral nanosystems for systemic siRNA delivery. Pharmacol Res, 2010, 62: 100-114

Open Access This article is distributed under the terms of the Creative Commons Attribution License which permits any use, distribution, and reproduction in any medium, provided the original author(s) and source are credited. 